\title{
Successful treatment of 5-fluorouracil mediated cardiotoxicity with uridine triacetate in a pediatric patient
}

\author{
Gisela Marrero-Rivera ${ }^{1}$, Cori Morrison ${ }^{2}$, Zachary LeBlanc ${ }^{2}$, Maria Velez ${ }^{2}$, and Dana \\ Leblanc $^{2}$ \\ ${ }^{1}$ Louisiana State University in New Orleans \\ ${ }^{2}$ Louisiana State University Health Sciences Center
}

September 24, 2020

\begin{abstract}
5-fluorouracil (5-FU) is a pyrimidine analogue used to treat adults with a variety of carcinomas but less frequently used in pediatrics. 5 -FU is a well-recognized cardiotoxic chemotherapy medications, particularly among adults with underlying cardiovascular disease, however this has rarely been reported in the pediatric population. We report a rare case of 5-FU induced cardiomyopathy in a pediatric patient with nasopharyngeal carcinoma (NPC), successfully managed with milrinone and uridine triacetate, underscoring the need for awareness and prompt recognition and medication administration for this potentially reversible condition.
\end{abstract}

Successful treatment of 5-fluorouracil mediated cardiotoxicity with uridine triacetate in a pediatric patient

Gisela Marrero-Rivera, $\mathrm{MD}^{1}$; Cori Morrison, $\mathrm{MD}^{1}$; Zachary LeBlanc, $\mathrm{MD}^{1}$; Maria Velez, $\mathrm{MD}^{1}$; Dana LeBlanc, $\mathrm{MD}^{1}$,

${ }^{1}$ Pediatric Hematology Oncology Department, Children's Hospital of New Orleans, Louisiana State University Health Sciences Center

\begin{tabular}{ll}
\hline Abbreviation & Full Term \\
\hline NPC & Nasopharyngeal Carcinoma \\
5-FU & 5-Fluorouracil \\
LV EF & Left Ventricle Ejection Fraction \\
FDA & Food and Drug Administration \\
COG & Children's Oncology Group \\
\hline
\end{tabular}

\section{Contact Information:}

Gisela Marrero-Rivera, MD FAAP

gmarre@lsuhsc.edu

504-896-9740; 787-314-4408

Word Count: 1,443

Keywords: Nasopharyngeal Carcinoma, 
5-Fluorouracil Induced Cardiomyopathy, Cardiotoxicity, Uridine Triacetate

\section{Successful treatment of 5-fluorouracil mediated cardiotoxicity with uridine triacetate in a pediatric patient}

Gisela Marrero-Rivera, MD; Cori Morrison, MD; Zachary LeBlanc, MD; Maria Velez, MD; Dana LeBlanc, $\mathrm{MD}$

\section{Summary :}

5 -fluorouracil (5-FU) is a pyrimidine analogue used to treat adults with a variety of carcinomas but less frequently used in pediatrics. This chemotherapy has known cardiotoxic side effects, particularly among adults with underlying cardiovascular disease, however this has rarely been reported in the pediatric population. We report a rare case of 5-FU induced cardiomyopathy in a pediatric patient with nasopharyngeal carcinoma (NPC), successfully managed with milrinone and uridine triacetate. This report underscores the need for prompt diagnosis and treatment of 5-FU induced cardiomyopathy.

\section{Introduction}

Nasopharyngeal carcinoma (NPC) is a very rare tumor in children. Although enormous differences exist among races and geographical groups, nasopharyngeal carcinoma makes up 1-5\% of all pediatric cancers and $20-50 \%$ of all primary malignant nasopharyngeal tumors in children. This disease has been linked to etiological factors such as Epstein Barr virus, consumption of food rich in nitrosamines, and genetic and epigenetic factors that are still unclear. Current treatment protocols use induction with Cisplatin-based chemotherapy and 5-FU with concomitant chemoradiotherapy. Prognosis with current protocols treating pediatric nasopharyngeal carcinoma is excellent with an overall survival higher than $85 \%$ [1].

5-FU is a known inducer of cardiotoxicity in adult patients, particularly those with underlying cardiovascular disease. The cardiotoxic effects of 5-FU in the pediatric population are not well described. Previous studies of adults with 5-FU-mediated cardiotoxicity described events of myocardial infarction, coronary vasospasm, arrhythmias or cardiomyopathy [2]. The mechanism is not fully understood; however, may be due to a combination of ischemia related to coronary vasospasm and direct myocardial cell toxicity [3]. Uridine triacetate was FDA approved in December of 2015 as a 5-FU reversal agent for the emergency treatment of patients with life-threatening toxicity.

\section{Case Report}

A 16-year-old female diagnosed with stage IVA NPC began treatment with a regimen of Cisplatin and 5FU per COG protocol ARAR0331. She presented with a one-year history of migraine headaches and left sided cranial nerve VII palsy secondary to tumor compression [Fig. 1]. She had no known underlying cardiovascular disease and an echocardiogram prior to the initiation of chemotherapy demonstrated normal cardiac function (left ventricular ejection fraction (LV EF) 68\%).

Planned initial chemotherapy regimen consisted of 3 cycles of Cisplatin $(80 \mathrm{mg} / \mathrm{m} 2 /$ day $)$ on day 1 and 5 -FU $(1,000 \mathrm{mg} / \mathrm{m} 2 /$ day continuous infusion) on days $1-4$. On day 3 of cycle 1 , she developed acute hypotension and tachycardia. Electrocardiogram demonstrated sinus tachycardia and echocardiogram showed significantly compromised cardiac function, with LV EF of 33\%. She was transferred to the pediatric ICU and milrinone infusion was initiated.

Cardiac function continued to decline over subsequent days [Fig. 2]. Due to the temporal relationship, 5-FU cardiotoxicity was considered as the etiology of the patient's cardiomyopathy. At hour 60 after the completion of 5-FU infusion, uridine triacetate therapy was initiated (10 grams every 6 hours orally for 5 days). Within 48 hours, LV EF improved to 48\%. By 96 hours, LV EF normalized and milrinone was weaned off. She completed the full regimen of uridine triacetate (total of 20 doses). The patient continued the chemotherapy regimen with cisplatin as monotherapy, and subsequently received external beam radiation therapy for local control. The patient responded to this therapy and remains in PET-negative remission 17 
months post-therapy. The most recent echocardiogram performed approximately 14 months following this event shows no evidence of cardiac dysfunction (LV EF 60\%).

\section{Discussion}

5-FU is a commonly used pyrimidine analog and antimetabolite in adult patients with solid tumors and has significant cardiotoxicity. There are currently only two cases of reported cardiotoxic effects of 5-FU in a pediatric setting. One author described a 14-year-old male with NPC who developed severe dilated cardiomyopathy after 5-FU infusion with reversal of cardiotoxicity after completion of treatment [4]. The other case was that of a 14-year-old male who experienced sudden death during the infusion of his fifth cycle of 5-FU, which was postulated to be due to arrhythmia and cardiomyopathy, though no autopsy was available. Interestingly, the patient in this case had experienced dilated cardiomyopathy with his first cycle of 5-FU that resolved. He tolerated cycles 2, 3 and 4 without issue, but experienced irreversible circulatory collapse during his fifth cycle [5]. Both of these case reports were published prior to the FDA approval of uridine triacetate.

Our case is the first to describe a female pediatric patient with 5-FU-mediated cardiotoxicity treated with uridine triacetate. She tolerated the antidote well and completed a 5-day course with mild diarrhea as the only adverse effect. Based on literature reports of potential adverse outcomes with repeated exposure to 5-FU, the treatment team elected to eliminate subsequent doses and instead proceeded with Cisplatin monotherapy. In addition, the patient received standard doses of external beam radiotherapy. Despite these necessary modifications to the treatment regimen, the patient has had continued reduction in the size of the residual mass on MRI and remains PET-negative >1-year post-therapy.

Uridine triacetate is FDA approved as a 5-FU reversal agent for the emergency treatment of patients with lifethreatening toxicity. Uridine triacetate is converted to uridine in circulation which competitively inhibits cell damage and cell death caused by 5-FU [6]. 5-FU-mediated cardiotoxicity may have a variety of presentations such as myocardial infarction, coronary vasospasm, arrhythmias or cardiomyopathy. The mechanism of 5-FUmediated cardiotoxicity is not fully understood; however, it may be due to a combination of ischemia related to coronary vasospasm and direct myocardial cell toxicity. Based on reports in the literature, cardiotoxicity appears to be more frequently associated with continuous infusion rather than bolus infusion, presumably due to a higher incidence of vasoconstrictive events with continuous infusion. Several cases of successful re-challenge with bolus 5-FU, utilizing calcium channel blockers (CCBs) and nitrates to prophylaxis against coronary vasospasm recurrence, have been reported in the literature [7]. However, since there is increased variability of time to symptom onset with the infusion of 5-FU, re-challenge with infusion of 5-FU has not been widely studied. Our patient's cardiotoxicity was fully reversed with the use of uridine triacetate due to prompt recognition of the condition as an adverse effect of 5-FU.

\section{Conclusion}

5-FU-mediated cardiotoxicity is life-threatening and underappreciated as a potential complication for pediatric patients. Prompt recognition and appropriate management is essential to achieve favorable outcomes, and normalization of cardiac function is possible following an event.

\section{Conflict of Interest:}

The authors declare no potential conflict of interest.

\section{References:}

1. González-Motta A, González G, Bermudéz Y, Maldonado MC, Castañeda JM, Lopéz D, Cotes-Mestre M. Pediatric Nasopharyngeal Cancer: Case Report and Review of the Literature. Cureus , 2016 8: e497.

2. Sara JD, Kaur J, Khodadadi R, Rehman M, Lobo R, Chakrabarti S, Herrmann J, Lerman A, Grothey A. 5-fluorouracil and cardiotoxicity: a review. Ther Adv Med Oncol , 2018. 
3. Fulbright JM. Review of Cardiotoxicity in Pediatric Cancer Patients: During and after Therapy. Cardiol Res Pract, 2011.

4. Blütters-Sawatzki R, Grathwohl J, Mertens R, Lampert F. Severe cardiotoxicity of high-dose 5-fluorouracil in combination with folinic acid, cisplatin and methotrexate in a 14-year-old boy with nasopharyngeal carcinoma (Schmincke tumor). Oncology , 1995 52: 291-294.

5. Radhakrishnan V, Bakhshi S. 5-Fluorouracil-induced Acute Dilated Cardiomyopathy in a Pediatric Patient. J Pediatr Hematol Oncol , 2011 33: 323.

6. Dj C, U M, Rj B, De B. Uridine Triacetate.. Hosp Pharm , 2016 51: 484-488.

7. Hansen RM, Ryan L, Anderson T, Krzywda B, Quebbeman E, Benson A, Haller DG, Tormey DC. Phase III study of bolus versus infusion fluorouracil with or without cisplatin in advanced colorectal cancer. J Natl Cancer Inst , 1996 88: 668-674.

8. DailyMed - VISTOGARD- uridine triacetate granule Available from: https://dailymed.nlm.nih.gov/dailymed/lookup.cfm?setid=5a0ba417-8a4a-4d7f-b85a-1839ee8bd3f1

Figure 1: Brain MRI with and without contrast at diagnosis showing a large left destructive mass at the left central skull base associated with the left cervical pathological lymph node.

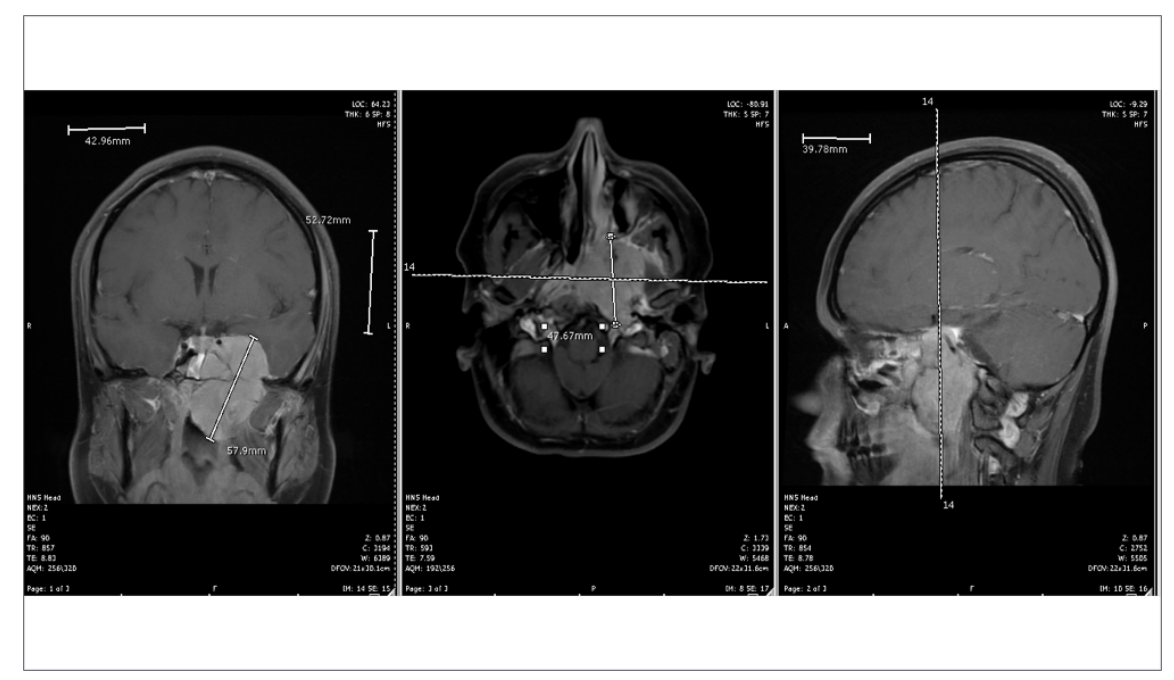

Figure 2 : Left Ventricular Ejection Fraction improvement with Uridine Triacetate treatment 


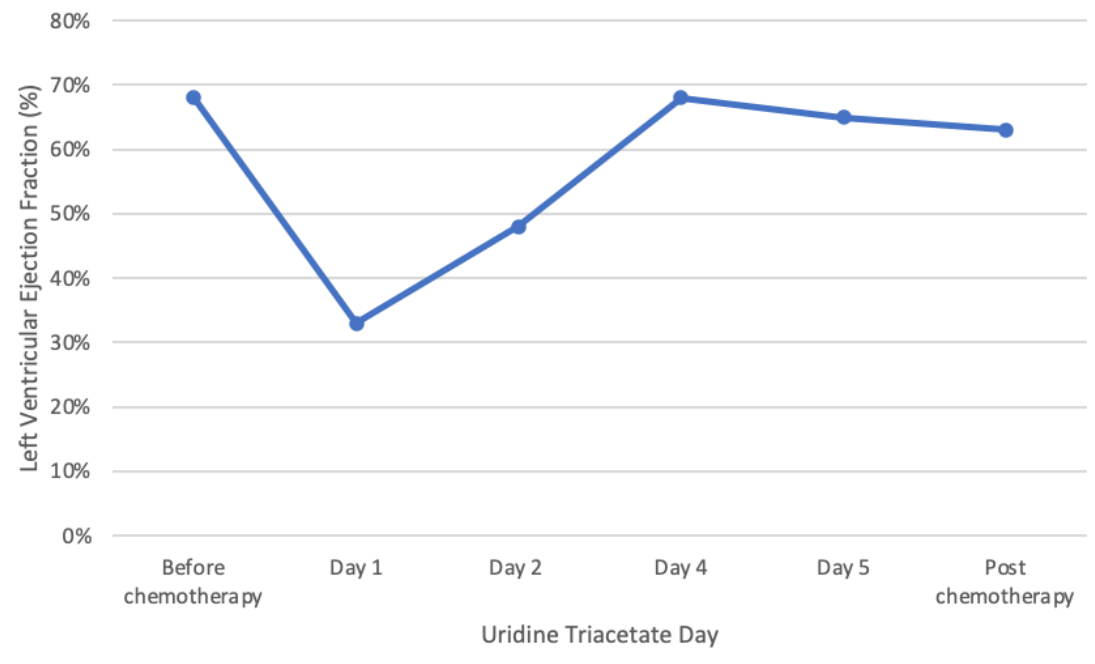


Figure 1: Brain MRI with and without contrast at diagnosis showing a large left destructive mass at the left central skull base associated with the left cervical pathological lymph node.

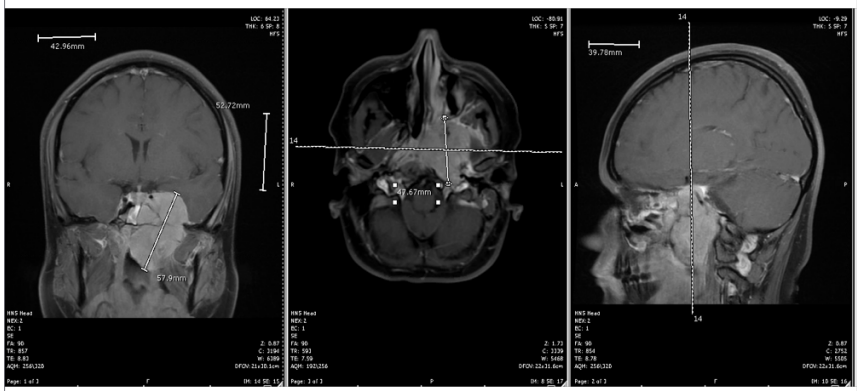


Figure 2: Left Ventricular Ejection Fraction improvement with Uridine Triacetate treatment

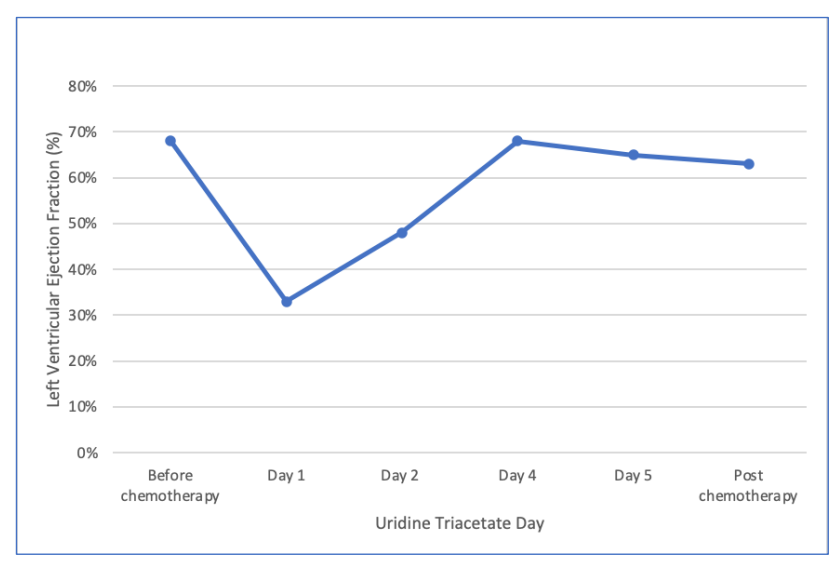

